\title{
Plasmons in Mesoscopic Gold Tapers
}

Wilfried Sigle ${ }^{1}$, Nahid Talebi ${ }^{1}$, Surong Guo ${ }^{1}$, Christian Knipl ${ }^{1}$, Christoph Lienau ${ }^{2}$, Martin Esmann $^{2}$, Simon F. Becker ${ }^{2}$, Ralf Vogelgesang ${ }^{2}$, Peter A. van Aken ${ }^{1}$

1. Max Planck Institute for Solid State Research, Stuttgart, Germany

2. Carl von Ossietzky University, Oldenburg, Germany

The ability of metallic nanostructures to sustain localized surface plasmons (LSPs) makes them essential elements of future nanophotonic devices. The optical fields associated with these LSPs are concentrated in volumes much smaller than allowed by the diffraction limit and the field distribution and resonance energies depend critically on the size and shape of the nanostructure. Of particular interest are structures that allow optical fields to be steered (waveguides) or concentrated and focused to a certain position. An additional requirement for applications is a broad bandwidth and low radiative losses of such structures. Some of these requirements can be met by metallic tapers [1,2].

An efficient tool for studying the plasmonic response of nanostructures is electron energy-loss spectroscopy (EELS). In EELS, the fast electron excites surface plasmons and, by passing the plasmonic electromagnetic field of the nanostructure, the electron loses energy. This energy loss can be measured with nanometer spatial resolution and sub-100 meV energy resolution. In our experiments we use the Zeiss SESAM microscope operated at $200 \mathrm{kV}$, equipped with the MANDOLINE energy filter and an omega-type electrostatic monochromator (CEOS, Germany). Data acquisition was performed in either EELS spectrum imaging or energy-filtering TEM (EFTEM) mode.

Gold tapers with very smooth surfaces were prepared at Oldenburg University by electrochemical etching in $\mathrm{HCl}$ [3]. The tapers were mounted on a 3-mm copper ring for TEM analysis. An annular darkfield image of a taper with $45^{\circ}$ opening angle is shown in Fig. 1(left). EELS spectra were recorded along the green arrow in aloof geometry. The spectra (Fig.1, right) exhibit pronounced maxima which blueshift as the electron beam approaches the apex. At the apex a very broadband spectrum is detected which demonstrates the ability of the taper to concentrate light of a wide range of wavelengths into a very small volume. These features are also visible in a series of EFTEM images (Fig. 2) where high field concentration at the apex is visible over a wide energy range and field maxima occur along the taper shaft.

Finite-difference time-domain calculations of 3D-tapers with an embedded relativistic electron source [4] were conducted in order to unravel the experimental results. We found that for tapers with sufficiently large opening angles the spectral maxima originate from phase matching between the electron field and the radiative taper modes [5]. The broad spectral feature at the apex is due to a rotationally symmetric (zero angular momentum) optical mode. Further experiments and calculations at different opening angles will be discussed. [6] 


\section{References:}

[1] M. I. Stockman, Phys. Rev. Lett. 93 (2004) p.137404.

[2] A. Bouhelier et al., J. Microsc. 210 (2002) p. 220.

[3] S. Schmidt et al., ACS Nano 6 (2012) p.6040.

[4] N. Talebi, New J. Phys. 16 (2014) p.053021.

[5] N. Talebi et al., ACS Nano 9 (2015) p.7641.

[6] The research leading to these results has received funding from the European Union Seventh Framework Programme [FP7/2007-2013] under grant agreement No. 312483 (ESTEEM2).

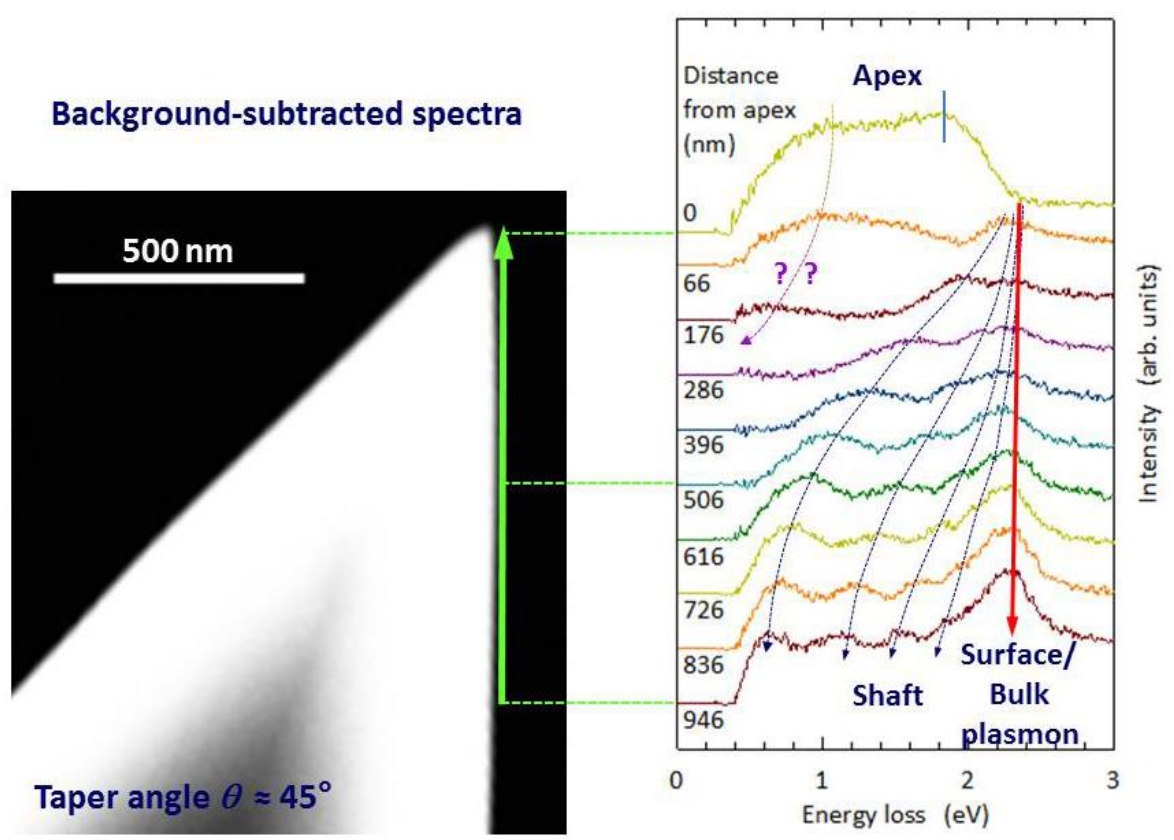

Figure 1. Left: HAADF image of a gold taper with $45^{\circ}$ opening angle. Right: Background-subtracted EELS spectra recorded along the taper shaft at different locations along the green arrow in the left image.

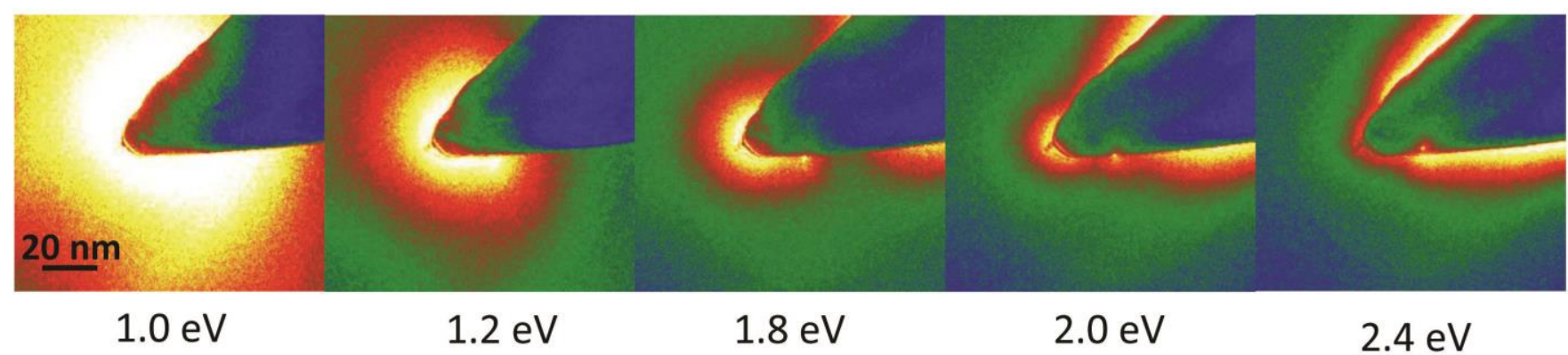

Figure 2. Series of EFTEM images recorded at different energy losses. Strong field enhancement at the tip is visible over a broad energy range. Along the shaft additional field maxima are visible. 